\title{
Lie Symmetry Analysis and Dynamics of Exact Solutions of the (2+1)-Dimensional Nonlinear Sharma-Tasso-Olver Equation
}

\author{
Sachin Kumar $\mathbb{D}^{1},{ }^{1}$ Ilyas Khan $\mathbb{D}^{2,3}$ Setu Rani, ${ }^{1}$ and Behzad Ghanbari ${ }^{4}$ \\ ${ }^{1}$ Department of Mathematics, Faculty of Mathematical Sciences, University of Delhi, Delhi-110007, India \\ ${ }^{2}$ Department of Mathematics, College of Science Al-Zulf, Majmaah University, Al-Majmaah 11952, Saudi Arabia \\ ${ }^{3}$ Faculty of Mathematics and Statistics, Ton Duc Thang University, Ho Chi Minh City 72915, Vietnam \\ ${ }^{4}$ Department of Engineering Science, Kermanshah University of Technology, Kermanshah, Iran \\ Correspondence should be addressed to Ilyas Khan; ilyaskhan@tdtu.edu.vn
}

Received 12 March 2021; Accepted 25 April 2021; Published 11 May 2021

Academic Editor: Fazal M. Mahomed

Copyright $\odot 2021$ Sachin Kumar et al. This is an open access article distributed under the Creative Commons Attribution License, which permits unrestricted use, distribution, and reproduction in any medium, provided the original work is properly cited.

In soliton theory, the dynamics of solitary wave solutions may play a crucial role in the fields of mathematical physics, plasma physics, biology, fluid dynamics, nonlinear optics, condensed matter physics, and many others. The main concern of this present article is to obtain symmetry reductions and some new explicit exact solutions of the $(2+1)$-dimensional Sharma-Tasso-Olver (STO) equation by using the Lie symmetry analysis method. The infinitesimals for the STO equation were achieved under the invariance criteria of Lie groups. Then, the two stages of symmetry reductions of the governing equation are obtained with the help of an optimal system. Meanwhile, this Lie symmetry method will reduce the STO equation into new partial differential equations (PDEs) which contain a lesser number of independent variables. Based on numerical simulation, the dynamical characteristics of the solitary wave solutions illustrate multiple-front wave profiles, solitary wave solutions, kink wave solitons, oscillating periodic solitons, and annihilation of parabolic wave structures via 3D plots.

\section{Introduction}

Most of the physical problems are inherently nonlinear because of their nature. These types of nonlinear problems are of much interest to engineers, mathematicians, physicists, and many other researchers because of their physical applications in scientific areas such as engineering, biology, theoretical physics, plasma physics, and condensed matter physics (see [1]). To predict the possible behaviors of physical phenomena, exact solutions of these nonlinear PDEs are very important. The various types of rational solutions were investigated by analytical and numerical methods such as the Lie symmetry method [2, 3], homogeneous balance method [4], tanh function method [5], Hirota bilinear method [6], Backlund transformation method [7], Darboux transformation method [8], the truncated Painleve expansion [9], and F-expansion method [10]. In solitary wave theory, a large variety of traveling waves are of much concern. Some important traveling waves are the kink waves and the solitons. The kink waves fall or rise from one asymptotic state to others, and the latter is the localized traveling waves which are asymptotically zero at large distances. Some potential applications of these wave solutions may be found in coastal areas and open oceans.

The $(1+1)$-dimensional STO equation is of the form

$$
u_{t}+\alpha u_{x}^{3}+\frac{3}{2} \alpha u_{x x}^{2}+\alpha u_{x x x}=0
$$

where $\alpha$ is some constant and the dissipative term $u_{x x x}$ provides damping of a small scale. A lot of researchers worked on the STO equation (1) due to its huge appearance in scientific applications. The STO equation is similar to the $\mathrm{KdV}$ equation and has been used to describe a wide range of physical phenomena of the evolution and interaction with the nonlinear waves, such as solitons and turbulence, fluid dynamics, continuum mechanics, and aerodynamics. The STO equation can also be used to describe how do nonlinear dispersive waves propagate in inhomogeneous media. It possesses the bi-Hamiltonian formulation and an infinite number of symmetries. Different nonlinear waves are 
described by the exact solutions for (1) with different forms. The generalized Kaup-Newell-type hierarchy of nonlinear evolution equations is related to the Sharma-Tasso-Olver equation from [11]. A large number of integrability properties of (1) are achieved in [12,13]. In [14], equation (1) was managed by Yan using the Cole-Hopf transformation method. By considering the improved tanh function method, fission and fusion for the Sharma-Tasso-Olver equation have some exact solutions in [15]. In ref. [16-18], some powerful techniques such as the Hirota direct method, extended hyperbolic function method, and Backlund transformation method are used for finding the explicit solutions of (1), respectively. Also, this equation was managed by using the tanh method and the extended tanh method (see [16]).

This research aims to find the exact-soliton solutions of $(2+1)$-dimensional STO equation. It is well known that the $(2+1)$-dimensional KP equation is an extended version of $(1+1)$-dimensional Korteweg-de Vries $(\mathrm{KdV})$ equation. Analogously, $(2+1)$-dimensional STO equation is an extended version of $(1+1)$-dimensional STO equation. The extended form of $(2+1)$-dimensional STO equation is introduced in [19], which is accomplished by adding a new term $u_{y y}$ in $(1+1)$-dimensional STO equation (1)

$$
\left(u_{t}+\alpha u_{x}^{3}+\frac{3}{2} \alpha u_{x x}^{2}+\alpha u_{x x x}\right)_{x}+\beta u_{y y}=0,
$$

where $\alpha$ and $\beta$ are two arbitrary constants and the unknown function $u$ depends on the spatial variables $x, y$ and temporal variable $t$. This equation encloses both nonlinear and linear terms. Using the tanh function method, the traveling wave solution of STO equation (2) is found as

$$
u(x, y, t)=c_{5} \mp \sqrt{6} \tanh \left(\mp \frac{x}{\sqrt{6}} \pm y c_{6} \pm \frac{1}{3} \sqrt{\frac{2}{3}} t\left(\alpha+9 c_{6}^{2} \beta\right)\right)
$$

where $c_{5}$ and $c_{6}$ are arbitrary constants. Here, we will solve a new $(2+1)$-dimensional equation using the similarity transformation method. Felix Klein (1849-1925) and M. Sophus Lie (1842-1899) introduced this method to construct integration theory for ODEs. For further details, one can refer to textbooks [20,21] or some articles [3, 22-27] as well as references therein. During this process, some variable transformations take place, which is used to obtain new solutions from the existing ones using differential operators, called the generators of the symmetry group. With the help of similarity forms, STM reduces the number of independent variables of the original PDE by one. As a result, the equation was reduced into a nonlinear PDE having one less independent variable. In the same way, one can reduce this nonlinear PDE into nonlinear ODE by reducing one independent variable, which can be solved mathematically.

Being motivated by the aforementioned references/ works, we would like to study to construct the explicit exactsoliton solutions by using the Lie symmetry approach. Besides, we derive various Lie symmetry reductions and localized solitary wave solutions for the considered equation.
Recently, Ben and Ma [19] investigated $(2+1)$-dimensional STO equation and found some rational solutions using the resulting trilinear form. Our analytic results in this paper are more general in terms of independent functions and entirely different from their works/findings. Furthermore, the physical behaviors of the soliton solutions are demonstrated through their evolution profiles.

The remaining manuscript is structured as follows. In Section 1, we discuss various algorithms for solving the STO equation and a new form of the equation by adding one term $u_{y y}$. The Lie symmetries, vector fields, infinitesimals, and an optimal system of one-dimensional subalgebra for the STO equation are depicted in Section 2. In Section 3, Lie symmetry reductions and analytical wave solutions are furnished. The dynamical analysis of attained soliton solutions is analyzed physically in Section 4. Lastly, a conclusion is drawn in Section 5.

\section{Lie Symmetry Analysis}

The motive of this present section is to describe the main steps used in obtaining the infinitesimal, Lie symmetries, and optimal system of one-dimensional subalgebra for STO equation (2). To find the invariant solutions of (2), the STM is applied. Since equation (2) is highly nonlinear, therefore to solve equation (2), we need to introduce one parameter $(\varepsilon)$ Lie group of infinitesimals transformation as

$$
\begin{aligned}
& \widetilde{x_{i}^{*}}=x_{i}+\varepsilon \xi_{i}(x, u)+O\left(\varepsilon^{2}\right), \\
& \widetilde{u^{*}}=u+\varepsilon \eta(x, u)+O\left(\varepsilon^{2}\right),
\end{aligned}
$$

where the infinitesimals $\xi_{1}=\xi^{(1)}, \xi_{2}=\xi^{(2)}, \xi_{3}=\tau$, and $\eta$ are the infinitesimals transformations for $x, y, t$, and $u$, respectively. The linked vector field $V$ with the above group of transformation is defined as

$$
V=\xi^{(1)} \frac{\partial}{\partial x}+\xi^{(2)} \frac{\partial}{\partial y}+\tau \frac{\partial}{\partial t}+\eta \frac{\partial}{\partial u}
$$

where the obtained functions $\xi^{(1)}, \xi^{(2)}, \tau$, and $\eta$ are found under the constraint:

$$
\begin{aligned}
\xi^{(1)} & =\left.\frac{\mathrm{d} \widetilde{x}}{\mathrm{~d} \varepsilon}\right|_{\varepsilon=0}, \\
\xi^{(2)} & =\left.\frac{\mathrm{d} \widetilde{y}}{\mathrm{~d} \varepsilon}\right|_{\varepsilon=0}, \\
\tau & =\left.\frac{\mathrm{d} \widetilde{t}}{\mathrm{~d} \varepsilon}\right|_{\varepsilon=0}, \\
\eta & =\left.\frac{\mathrm{d} \widetilde{u}}{\mathrm{~d} \varepsilon}\right|_{\varepsilon=0} .
\end{aligned}
$$

If equation (2) is symmetrical about the vector field (5), then $V$ must fulfill the condition

$$
\left.\operatorname{Pr}^{(4)} V(\Delta)\right|_{\Delta=0}=0,
$$

where $P r^{(4)} V$ represents the fourth-order prolongation and is given by [21] 


$$
\begin{aligned}
V^{(4)}= & V+\eta^{x} \frac{\partial}{\partial u_{x}}+\eta^{y} \frac{\partial}{\partial u_{y}}+\eta^{t} \frac{\partial}{\partial u_{t}}+\eta^{x x} \frac{\partial}{\partial u_{x x}}+\eta^{x t} \frac{\partial}{\partial u_{x t}}+\cdots+\eta^{y y} \frac{\partial}{\partial u_{y y}}+\cdots \eta^{t t} \frac{\partial}{\partial u_{t t}} \\
& +\eta^{x x x} \frac{\partial}{\partial u_{x x x}}+\eta^{x x y} \frac{\partial}{\partial u_{x x y}} \cdots+\eta^{y y y} \frac{\partial}{\partial u_{y y y}}+\cdots+\eta^{t t t} \frac{\partial}{\partial u_{t t t}}+\eta^{x x x x} \frac{\partial}{\partial u_{x x x x}}+\eta^{x x x t} \frac{\partial}{\partial u_{x x x t}}+\cdots+\eta^{y y y y} \frac{\partial}{\partial u_{y y y}}+\cdots+\eta^{t t t t} \frac{\partial}{\partial u_{t t t t}}
\end{aligned}
$$

Applying the above prolongation formula to (2) under where invariance condition, the symmetry condition is

$$
\begin{aligned}
\eta^{x t} & +3 \alpha u_{x}^{2} \eta^{x x}+6 \alpha u_{x} u_{x x} \eta^{x}+3 \alpha u_{x x x} \eta^{x x}+3 \alpha u_{x x} \eta^{x x x} \\
& +\alpha \eta^{x x x x}+\beta \eta^{y y}=0,
\end{aligned}
$$

$$
\begin{aligned}
\eta^{x} & =D_{x}(\eta)-u_{x}\left(D_{x} \xi^{(1)}\right)-u_{y}\left(D_{x} \xi^{(2)}\right)-u_{t}\left(D_{x} \tau\right), \\
\eta^{y} & =D_{y}(\eta)-u_{x}\left(D_{y} \xi^{(1)}\right)-u_{y}\left(D_{y} \xi^{(2)}\right)-u_{t}\left(D_{y} \tau\right), \\
\eta^{x x} & =D_{x}\left(\eta^{x}\right)-u_{x x}\left(D_{x} \xi^{(1)}\right)-u_{x y}\left(D_{x} \xi^{(2)}\right)-u_{x t}\left(D_{x} \tau\right), \\
\eta^{x t} & =D_{t}\left(\eta^{x}\right)-u_{x x}\left(D_{t} \xi^{(1)}\right)-u_{x y}\left(D_{t} \xi^{(2)}\right)-u_{x t}\left(D_{t} \tau\right), \\
\eta^{y y} & =D_{y}\left(\eta^{y}\right)-u_{y x}\left(D_{y} \xi^{(1)}\right)-u_{y y}\left(D_{y} \xi^{(2)}\right)-u_{y t}\left(D_{y} \tau\right), \\
\eta^{x x x} & =D_{x}\left(\eta^{x x}\right)-u_{x x x}\left(D_{x} \xi^{(1)}\right)-u_{x x y}\left(D_{x} \xi^{(2)}\right)-u_{x x t}\left(D_{x} \tau\right), \\
\eta^{x x x x} & =D_{x}\left(\eta^{x x x}\right)-u_{x x x x}\left(D_{x} \xi^{(1)}\right)-u_{x x x y}\left(D_{x} \xi^{(2)}\right)-u_{x x x t}\left(D_{x} \tau\right)
\end{aligned}
$$

are the coefficients of $\operatorname{Pr}^{(4)} V(\Delta)=0$ and $D_{x}, D_{y}$, and $D_{t}$ are total derivative operators that can be expressed as in [28].

$$
D_{i}=\frac{\partial}{\partial x_{i}}+u_{i} \frac{\partial}{\partial u}+u_{i j} \frac{\partial}{\partial u_{j}}+\cdots+, \quad \text { for } i=1,2,3,4 \text {. }
$$

Using (10) and (9), then the determining equations

$$
\begin{aligned}
\left(\eta_{u}\right)_{u} & =\left(\eta_{u}\right)_{x}=\left(\eta_{u}\right)_{y y}=0, \\
\left(\xi_{t}\right)_{t} & =\left(\xi_{t}\right)_{u}=\left(\xi_{t}\right)_{x}=\left(\xi_{t}\right)_{y}=0, \\
\left(\xi_{x}\right)_{t} & =\left(\xi_{x}\right)_{u}=\left(\xi_{x}\right)_{x}=0, \\
\left(\xi_{x}\right)_{y} & =\frac{-\left(\xi_{y}\right)_{t}}{2 \beta}, \\
\left(\xi_{y}\right)_{u} & =\left(\xi_{y}\right)_{x}=\left(\xi_{y}\right)_{y}=\left(\xi_{y}\right)_{t t}=0 .
\end{aligned}
$$

On solving equation (12), we obtain infinitesimal generators as

$$
\begin{gathered}
\xi^{(1)}=-\frac{c_{1} y}{2 \beta}+c_{4}, \quad \xi^{(2)}=c_{1} t+c_{2}, \\
\tau=c_{3}, \quad \eta=f_{1}(t) y+f_{2}(t),
\end{gathered}
$$

where $c_{i} ; 1 \leq i \leq 4$ are arbitrary constants and $f_{1}(t)$ and $f_{2}(t)$ are functions obtained in the processing of infinitesimals. The choice of $f_{i}^{\prime} s$ can achieve some physical structures of solutions of equation (2). The symmetry algebra of equation (2) can be obtained by the given vectors

$$
\begin{aligned}
& V_{1}=-\frac{y}{2 \beta} \frac{\partial}{\partial x}+t \frac{\partial}{\partial y}, \\
& V_{2}=\frac{\partial}{\partial y}, \\
& V_{3}=\frac{\partial}{\partial t}, \\
& V_{4}=\frac{\partial}{\partial x}, \\
& V_{5}=y f_{1}(t) \frac{\partial}{\partial u}, \\
& V_{6}=f_{2}(t) \frac{\partial}{\partial u},
\end{aligned}
$$

such that $V=c_{1} V_{1}+c_{2} V_{2}+c_{3} V_{3}+c_{4} V_{4}+V_{5}\left(f_{1}\right)+V_{6}$ $\left(f_{2}\right)$.

It has been noted from the commutative relations of vector fields that the Lie group of transformations formed an infinite-dimensional Lie algebra with functions $f_{1}(t)$ and $f_{2}(t)$ and its commutation table (Table 1 ) is skew-symmetric with each diagonal entry zero. 
TABLE 1: Commutative table.

\begin{tabular}{lcccccc}
\hline$\left[V_{i} V_{j}\right]$ & $V_{1}$ & $V_{2}$ & $V_{3}$ & $V_{4}$ & $V_{5}$ & $V_{6}$ \\
\hline$V_{1}$ & 0 & $\left(V_{4} / 2 \beta\right)$ & $-V_{2}$ & 0 & $t\left(f_{1}(t) / f_{2}(t)\right) V_{6}$ & 0 \\
$V_{2}$ & $-\left(V_{4} / 2 \beta\right)$ & 0 & 0 & 0 & $\left(f_{1}(t) / f_{2}(t)\right) V_{6}$ & 0 \\
$V_{3}$ & $V_{2}$ & 0 & 0 & 0 & $\left(f_{1}^{\prime}(t) / f_{1}(t)\right) V_{5}$ & $\left(f_{2}^{\prime}(t) / f_{2}(t)\right) V_{6}$ \\
$V_{4}$ & 0 & 0 & 0 & 0 & 0 & 0 \\
$V_{5}$ & $-t\left(f_{1}(t) / f_{2}(t)\right) V_{6}$ & $-\left(f_{1}(t) / f_{2}(t)\right) V_{6}$ & $-\left(f_{1}^{\prime}(t) / f_{1}(t)\right) V_{5}$ & 0 & 0 & 0 \\
$V_{6}$ & 0 & 0 & $-\left(f_{2}^{\prime}(t) / f_{2}(t)\right) V_{6}$ & 0 & 0 \\
\hline
\end{tabular}

The Lie series to calculate the adjoint relation is furnished as

$$
\operatorname{Ad}\left(\exp \left(\varepsilon V_{i}\right)\right) V_{j}=V_{j}-\varepsilon\left[V_{i}, V_{j}\right]+\frac{\varepsilon^{2}}{2}\left[V_{i},\left[V_{i}, V_{j}\right]\right]+\cdots .
$$

Furthermore, an optimal system of one-dimensional subalgebra is achieved by means of Olver's technique [21]. The desired optimal system for STO equation (2) is obtained by using adjoint table (Table 2). Following is the given onedimensional subalgebras for STO equation (2):

$$
\begin{aligned}
& \mathscr{T}_{1}=V_{2}+V_{4}, \\
& \mathscr{T}_{2}=V_{2}+V_{3}+V_{4}, \\
& \mathscr{T}_{3}=V_{4}+V_{5}, \\
& \mathscr{T}_{4}=V_{4}+V_{6}, \\
& \mathscr{T}_{5}=c_{1} V_{1}+c_{2} V_{2}+c_{3} V_{3}+c_{4} V_{4}+V_{5}+V_{6}, \\
& \mathscr{T}_{6}=c_{2} V_{2}+c_{3} V_{3}+c_{4} V_{4}+V_{5}+V_{6} .
\end{aligned}
$$

\section{Symmetry Reductions and Explicit Solutions}

In this section, we will construct some exact-soliton solutions with the aid of 1-dimensional optimal system of symmetry subalgebras. To achieve exact-invariant solutions for the considered equation (2), the corresponding Lagrange's system is chosen as

$$
\frac{\mathrm{d} x}{\xi^{(1)}}=\frac{\mathrm{d} y}{\xi^{(2)}}=\frac{\mathrm{d} t}{\tau}=\frac{\mathrm{d} u}{\eta}
$$

3.1. Subalgebra $\mathscr{T}_{1}=V_{2}+V_{4}=(\partial / \partial y)+(\partial / \partial x)$. Using vector field $V_{2}+V_{4}$, equation (2) is turned into

$$
\alpha U_{X X X X}+3 \alpha U_{X X} U_{X X X}+3 \alpha U_{X X} U_{X}^{2}+\beta U_{X X}+U_{X T}=0 \text {, }
$$

where $u(x, y, t)=U(X, T)$ and $X=x-y, T=t$.

Since equation (18) is also a nonlinear PDE and contains one dependent and two independent variables, we will again apply STM on this equation to construct new infinitesimal generators of the form

$$
\begin{aligned}
\xi_{X} & =k_{2}, \\
\xi_{T} & =k_{1}, \\
\eta_{U} & =g_{1}(T),
\end{aligned}
$$

where $k_{1}$ and $k_{2}$ are arbitrary constants and $g_{1}(T)$ is an arbitrary function. Then, the required characteristic equation is

$$
\frac{\mathrm{d} X}{k_{2}}=\frac{\mathrm{d} T}{k_{1}}=\frac{\mathrm{d} U}{g_{1}(T)}
$$
form

On solving equation (20), we get the following similarity

$$
U(X, T)=\frac{1}{k_{1}} \int g_{1}(T) \mathrm{d} T+U_{1}\left(X_{1}\right),
$$

where $X_{1}=X-\left(k_{2} / k_{1}\right) T$. By using (21) into (18), then reduced form of ODE is

$$
\alpha k_{1} U_{1}^{\prime \prime \prime}+3 \alpha k_{1} U_{1}^{\prime \prime} U_{1}^{\prime \prime}+3 \alpha k_{1} U_{1}^{\prime 2} U_{1}^{\prime \prime}+\left(k_{1} \beta-k_{2}\right) U_{1}^{\prime \prime}=0,
$$

which on integration gives

$$
\alpha k_{1} U_{1}^{\prime \prime}+\frac{3}{2} \alpha k_{1} U_{1}^{\prime \prime 2}+\alpha k_{1} U_{1}^{\prime 3}+\left(k_{1} \beta-k_{2}\right) U_{1}^{\prime}=C
$$

where $C$ is constant of integration and, represents the derivative of $U_{1}$ w.r.t $X_{1}$. One of the particular solutions of equation (23) is

$$
U_{1}\left(X_{1}\right)=a_{0}-X_{1}\left(\frac{T}{3 \sqrt{[3]} 2 \alpha k_{1}}+\frac{\sqrt{[3]} 2\left(k_{2}-\beta k_{1}\right)}{T}\right),
$$

where

$T=\sqrt{[3]} \sqrt{729 \alpha^{4} C^{2} k_{1}^{6}-108 \alpha^{3} k_{1}^{3}\left(k_{2}-\beta k_{1}\right)^{3}}-27 \alpha^{2} C k_{1}^{3}$. as

Hence, the exact explicit solution of equation (2) is given

$$
u(x, y, t)=a_{0}+\left(\frac{T}{3 \sqrt{[3]} 2 \alpha k_{1}}+\frac{\sqrt{[3]} 2\left(k_{2}-\beta k_{1}\right)}{T}\right)\left(\frac{k_{2} t}{k_{1}}-x+y\right)+\frac{\int g_{1}(t) \mathrm{d} t}{k_{1}} .
$$


Table 2: Adjoint table.

\begin{tabular}{lcccccc}
\hline [Ad] & $V_{1}$ & $V_{2}$ & $V_{3}$ & $V_{4}$ & $V_{5}$ & $V_{6}$ \\
\hline$V_{1}$ & $V_{1}$ & $V_{2}-\varepsilon\left(V_{4} / 2 \beta\right)$ & $V_{3}+\varepsilon V_{2}$ & $V_{4}$ & $V_{5}-\varepsilon\left(t f_{1}(t) / f_{2}(t)\right) V_{6}$ & $V_{6}$ \\
$V_{2}$ & $V_{1}+\varepsilon\left(V_{4} / 2 \beta\right)$ & $V_{2}$ & $V_{3}$ & $V_{4}$ & $V_{5}-\varepsilon\left(f_{1}(t) / f_{2}(t)\right) V_{6}$ & $V_{6}$ \\
$V_{3}$ & $V_{1}-\varepsilon V_{2}$ & $V_{2}$ & $V_{3}$ & $V_{4}$ & $V_{5}-\varepsilon\left(f_{1}^{\prime}(t) / f_{1}(t)\right) V_{5}$ & $V_{6}-\varepsilon\left(f_{2}^{\prime}(t) / f_{2}(t)\right) V_{6}$ \\
$V_{4}$ & $V_{1}$ & $V_{2}$ & $V_{3}$ & $V_{4}$ & $V_{5}$ & $V_{6}$ \\
$V_{5}$ & $V_{1}+\varepsilon\left(t f_{1}(t) / f_{2}(t)\right) V_{6}$ & $V_{2}+\varepsilon\left(f_{1}(t) / f_{2}(t)\right) V_{6}$ & $V_{3}+\varepsilon\left(f_{1}^{\prime}(t) / f_{1}(t)\right) V_{5}$ & $V_{4}$ & $V_{5}$ & $V_{6}$ \\
$V_{6}$ & $V_{1}$ & $V_{2}$ & $V_{3}+\varepsilon\left(f_{2}^{\prime}(t) / f_{2}(t)\right) V_{6}$ & $V_{4}$ & $V_{5}$ & $V_{6}$ \\
\hline
\end{tabular}

3.2. Subalgebra $\mathscr{T}_{2}=V_{2}+V_{3}+V_{4}=(\partial / \partial y)+(\partial / \partial t)+$ $(\partial / \partial x)$. For $V_{2}+V_{3}+V_{4}$, equation (2) is converted into

$$
\beta U_{Y Y}+U_{X X}\left(-1+3 \alpha\left(U_{X}^{2}+U_{X X X}\right)\right)+\alpha U_{X X X X}-U_{X Y}=0
$$

where $u(x, y, t)=U(X, Y)$ with $X=x-t, Y=y-t$. Again applying STM on equation (26), obtained form of infinitesimals is

$$
\xi_{X}=k_{4}, \quad \xi_{Y}=k_{3}, \quad \eta_{U}=k_{5} Y+k_{6} .
$$

where $k_{3}, k_{4}, k_{5}$, and $k_{6}$ are constants. Then, the associated Lagrange's system is found as

$$
\frac{\mathrm{d} X}{k_{4}}=\frac{\mathrm{d} T}{k_{3}}=\frac{\mathrm{d} U}{k_{5} Y+k_{6}} .
$$

On solving equation (28), we get a new similarity form of $U$ as

$$
U(X, Y)=\frac{\left(\left(k_{5} Y^{2}\right) / 2\right)+k_{6} Y}{K_{3}}+U_{2}\left(X_{2}\right),
$$

with $X_{2}=X-\left(k_{4} / k_{3}\right) Y$ as a similarity variable. By using equation (29) into (26), we get an ODE of the form

$$
\alpha k_{3}^{2} U_{2}^{\prime \prime}+U_{2}^{\prime \prime}\left(\beta k_{4}^{2}+k_{4} k_{3}-k_{3}^{2}+3 \alpha k_{3}^{2}\left(U_{2}^{\prime \prime}+U_{2}^{\prime 2}\right)\right)+\beta k_{3} k_{5}=0 .
$$

Since equation (30) is tedious nonlinear ODE, therefore by taking $k_{5}=0$ in equation (30), we attain one particular solution of the type

$$
U_{2}\left(X_{2}\right)=b_{1} X_{2}+b_{0}
$$

where $b_{0}$ and $b_{1}$ are arbitrary constants. Thus, the explicit exact solution for STO equation (2) is

$$
u(x, y, t)=b_{1}\left(-\frac{k_{4}(y-t)}{k_{3}}-t+x\right)+b_{0}+\frac{k_{6}(y-t)}{k_{3}} .
$$

3.3. Subalgebra $\mathscr{T}_{3}=V_{4}+V_{5}=(\partial / \partial x)+y f_{1}(t)(\partial / \partial u)$. Using vector field $V_{4}+V_{5}$, equation (2) is transformed into

$$
\beta U_{Y Y}+Y f_{1}^{\prime}(T)=0
$$

with $u(x, y, t)=x Y f_{1}(T)+U(Y, T)$ and $Y=y, T=t$. The invariant solution of PDE (33) is

$$
U(Y, T)=-\frac{Y^{3} f_{1}^{\prime}(t)}{6 \beta}+Y h_{1}(T)+h_{2}(T) .
$$
is

Therefore, the explicit exact solution of STO equation (2)

$$
u(x, y, t)=-\frac{y^{3} f_{1}^{\prime}(t)}{6 \beta}+x y f_{1}(t)+y h_{1}(t)+h_{2}(t) .
$$

3.4. Subalgebra $\mathscr{T}_{4}=V_{4}+V_{6}=(\partial / \partial x)+f_{2}(t)(\partial / \partial u)$. Using $V_{4}+V_{6}$, then, symmetry reduction is easily obtained as

$$
\beta U_{Y Y}+f_{2}^{\prime}(T)=0
$$

where $u(x, y, t)=x f_{2}(T)+U(Y, T)$ with $Y=y, T=t$. Thus, reduced PDE has the solution

$$
U(Y, T)=-\frac{Y^{2} f_{2}^{\prime}(t)}{2 \beta}+Y h_{1}(T)+h_{2}(T) .
$$

Thus, leading analytic solution for the STO equation is

$$
u(x, y, t)=-\frac{y^{2} f_{2}^{\prime}(t)}{2 \beta}+x f_{2}(t)+y h_{1}(t)+h_{2}(t) .
$$

3.5. Subalgebra $\mathscr{T}_{5}=c_{1} V_{1}+c_{2} V_{2}+c_{3} V_{3}+c_{4} V_{4}+V_{5}+$ $V_{6}=-\left(c_{1} y / 2 \beta\right)(\partial / \partial x)+c_{1} t(\partial / \partial y)+c_{2}(\partial / \partial y)+c_{3}(\partial / \partial t)+$ $c_{4}(\partial / \partial x)+y f_{1}(t)(\partial / \partial u)+f_{2}(t)(\partial / \partial u)$. Using (13) and (17), we will get the following Lagrange system:

$$
\frac{\mathrm{d} x}{-\left(c_{1} y / 2 \beta\right)+c_{4}}=\frac{\mathrm{d} y}{c_{1} t+c_{2}}=\frac{\mathrm{d} t}{c_{3}}=\frac{\mathrm{d} u}{f_{1}(t) y+f_{2}(t)} .
$$

It follows the similarity form as

$$
u(x, y, t)=\int \frac{\left(y f_{1}(t)+f_{2}(t)\right)}{c_{3}} \mathrm{~d} t+U(X, Y),
$$

where

$$
\begin{aligned}
& X=x+\frac{3 c_{1} c_{2} t^{2}+c_{1}^{2} t^{3}+6 c_{1} c_{3} t Y-12 c_{3} c_{4} t \beta}{12 c_{3}^{2} \beta}, \\
& Y=y-\frac{c_{2} t}{c_{3}}+\frac{c_{1} t^{2}}{2 c_{3}}
\end{aligned}
$$

are similarity variables with $U(X, Y)$ as a similarity function. Insert the above value of $u(x, y, t)$ from (40) into STO equation (2). Then, the reduced equation is given as 


$$
2 c_{3} \alpha \beta U_{X X X X}+2 c_{3} \beta^{2} U_{Y Y}-2 c_{2} \beta U_{X Y}+U_{X X}\left(c_{1} Y-2 c_{4} \beta+6 c_{3} \alpha \beta\left(U_{X}^{2}+U_{X X X}\right)\right)=0
$$
as

Again applying STM, equation (42) admits infinitesimals

$$
\begin{aligned}
\widehat{\xi^{1}} & =a_{1}, \\
\widehat{\xi^{2}} & =0, \\
\widehat{\eta} & =a_{2} Y+a_{3},
\end{aligned}
$$

where $a_{i} ; 1 \leq i \leq 3$ are arbitrary constants.

3.5.1. If $a_{2} \neq 0$ in Equation (43). Then, the characteristic equation is as follows:

$$
\frac{\mathrm{d} X}{A_{1}}=\frac{\mathrm{d} Y}{0}=\frac{\mathrm{d} U}{Y+A_{3}},
$$

where $A_{1}=\left(a_{1} / a_{2}\right)$ and $A_{3}=\left(a_{3} / a_{2}\right)$. Integration of equation (44) yields the following form of $U$

$$
U(X, Y)=\frac{X Y}{A_{1}}+\frac{X A_{3}}{A_{1}}+U_{3}\left(X_{3}\right),
$$

where $X_{3}=Y$. Using (45) into (42), we get an ODE as

$$
U_{3}^{\prime \prime}\left(X_{3}\right)=\frac{c_{2}}{A_{1} c_{3} \beta} \text {. }
$$

The solution of ODE (46) is given as

$$
U_{3}\left(X_{3}\right)=\frac{c_{2} X_{3}^{2}}{2 A_{1} c_{3} \beta}+B X_{3}+A,
$$

where $A$ and $B$ are integrating constants. On comparing equations (40), (45), and (47), the explicit solution for equation (2) is obtained as

$$
\begin{aligned}
u(x, y, t)= & \frac{1}{12 A_{1} c_{3}^{3} \beta}\left[\left(2 c_{2} t+c_{1} t^{2}-2 c_{3} y\right)\left(3 c_{2}^{2} t+3 c_{1} c_{2} t^{2}+c_{1}^{2} t^{3}-3 c_{2} c_{3} y-3 c_{1} c_{3} t y+6 c_{3}\left(c_{4} t-c_{3} x\right) \beta\right)\right. \\
& +A_{3} c_{3}\left(c_{1} t\left(-3 c_{2} t-2 c_{1} t^{2}+6 c_{3} y\right)+12 c_{3}\left(-c_{4} t+c_{3} x\right) \beta\right)+6 A_{1} c_{3}^{2} \beta\left(-t\left(2 c_{2}+c_{1} t\right) B+2 c_{3}(A+y B)+2 \int y f_{1}(t)\right. \\
& \left.\left.+f_{2}(t) \mathrm{d} t\right)\right]
\end{aligned}
$$

Taking the appropriate choice of $A=1$ and $B=2 \sqrt{\left(c_{2} /\left(2 A_{1} c_{3} \beta\right)\right)}$ in equation (47), we get

$$
U_{3}\left(X_{3}\right)=\left(\sqrt{\frac{c_{2}}{2 a_{1} c_{3} \beta}} X_{3}+1\right)^{2} \text {. }
$$

Thus, from equations (40), (45), and (49), the leading solution is of the form

$$
\begin{aligned}
u(x, y, t)= & \frac{1}{12 A_{1} c_{3}^{3} \beta}\left[\left(2 c_{2} t+c_{1} t^{2}-2 c_{3} y\right)\left(3 c_{2}^{2} t+3 c_{1} c_{2} t^{2}+c_{1}^{2} t^{3}-3 c_{2} c_{3} y-3 c_{1} c_{3} t y+6 c_{3}\left(c_{4} t-c_{3} x\right) \beta\right)\right. \\
& +A_{3} c_{3}\left(c_{1} t\left(-3 c_{2} t-2 c_{1} t^{2}+6 c_{3} y\right)+12 c_{3}\left(-c_{4} t+c_{3} x\right) \beta\right) \\
& \left.+6 A_{1} c_{3}^{2} \beta\left(-\sqrt{2} t\left(2 c_{2}+c_{1} t\right) \sqrt{\frac{c_{2}}{c_{3} \beta A_{1}}}+2\left(c_{3}+\sqrt{2} c_{3} y \sqrt{\frac{c_{2}}{c_{3} \beta A_{1}}}\right)+2 \int y f_{1}(t)+f_{2}(t) \mathrm{d} t\right)\right]
\end{aligned}
$$

3.5.2. If $a_{2}=0$ in Equation (43). Then, from (43), the Lagrange equation takes the form

$$
\frac{\mathrm{d} X}{a_{1}}=\frac{\mathrm{d} Y}{0}=\frac{\mathrm{d} U}{a_{3}} .
$$

Therefore, the similarity form is

$$
U(X, Y)=\frac{a_{3} X}{a_{1}}+U_{4}\left(X_{4}\right)
$$

where $X_{4}=Y$ and $U_{4}\left(X_{4}\right)$ are the similarity variable and similarity function, respectively. Substituting equation (52) in (42), one gets the following ODE: 


$$
U_{4}^{\prime \prime}\left(X_{4}\right)=0 .
$$

On solving equation (53),

$$
U_{4}\left(X_{4}\right)=C X_{4}+D,
$$

$$
\begin{aligned}
u(x, y, t)= & \frac{1}{12 a_{1} c_{3}^{2} \beta}\left(a_{3} c_{1} t\left(-3 c_{2} t-2 c_{1} t^{2}+6 c_{3} y\right)+6 c_{3}\left(-2 a_{3} c_{4} t-a_{1} t C\left(2 c_{2}+c_{1} t\right)+2 a_{3} c_{3} x+2 a_{1} c_{3}(C y+D)\right) \beta\right) \\
& +\int \frac{y f_{1}(t)+f_{2}(t)}{c_{3}} \mathrm{~d} t
\end{aligned}
$$

3.6. Subalgebra $\mathscr{T}_{6}=c_{2} V_{2}+c_{3} V_{3}+c_{4} V_{4}+V_{5}+V_{6}=c_{2}$ $(\partial / \partial y)+c_{3}(\partial / \partial t)+c_{4}(\partial / \partial x)+y f_{1}(t)(\partial / \partial u)+f_{2}(t)(\partial / \partial u)$. The required characteristic equation is

$$
\frac{\mathrm{d} x}{c_{4}}=\frac{\mathrm{d} y}{c_{2}}=\frac{\mathrm{d} t}{c_{3}}=\frac{\mathrm{d} u}{y f_{1}(t)+f_{2}(t)} .
$$

Solution of (56) predicts the similarity function

$$
u(x, y, t)=U(X, Y)+\int \frac{y f_{1}(t)+f_{2}(t)}{c_{3}} \mathrm{~d} t,
$$

with similarity variables $X=x-\left(c_{4} t / c_{3}\right)$ and $Y=$ $y-\left(c_{2} t / c_{3}\right)$. Incorporating equation (57) into equation (2) yields a PDE of the form

$$
\begin{aligned}
c_{3} \beta U_{Y Y} & +3 c_{3} \alpha U_{X X} U_{X}^{2}+3 c_{3} \alpha U_{X X} U_{X X X}+c_{3} \alpha U_{X X X X} \\
& -c_{2} U_{X Y}-c_{4} U_{X X}=0 .
\end{aligned}
$$

By applying the STM on (58), we get a new set of infinitesimals as

$$
\begin{aligned}
\widehat{\xi^{1}} & =a_{2}, \\
\widehat{\xi}^{2} & =a_{1}, \\
\widehat{\eta} & =a_{3} Y+a_{4},
\end{aligned}
$$

where $a_{i} ; 1 \leq i \leq 4$ are arbitrary constants. The Lagrange form for (59) reads

$$
\frac{\mathrm{d} X}{a_{2}}=\frac{\mathrm{d} Y}{a_{1}}=\frac{\mathrm{d} U}{a_{3} Y+a_{4}} .
$$

3.6.1. If $a_{3} \neq 0$ in Equation (59). Then, we have

$$
\frac{\mathrm{d} X}{B_{2}}=\frac{\mathrm{d} Y}{B_{1}}=\frac{\mathrm{d} U}{Y+B_{3}},
$$

where $B_{1}=\left(a_{1} / a_{3}\right), B_{2}=\left(a_{2} / a_{3}\right)$, and $B_{3}=\left(a_{4} / a_{3}\right)$. To get the solution of (2), the new similarity form of (61) is

$$
U(X, Y)=\frac{Y^{2}}{2 B_{1}}+\frac{B_{3} Y}{B_{1}}+U_{5}\left(X_{5}\right),
$$

where $C$ and $D$ are called integrating constants. From (40), (52), and (54), we will obtain the analytic solution of the STO equation with $X_{5}=X-\left(B_{2} Y / B_{1}\right)$ as the similarity variable. Using the value of (62) into (58), we get an ODE of the form

$$
\begin{aligned}
c_{3} \alpha B_{1}^{2} U_{5}^{\prime \prime} & +3 c_{3} \alpha B_{1}^{2} U_{5}^{\prime \prime} U_{5}^{\prime \prime}+3 c_{3} \alpha B_{1}^{2} U_{5}^{\prime 2} U_{5}^{\prime \prime}-c_{4} B_{1}^{2} U_{5}^{\prime \prime} \\
& +c_{2} B_{1} B_{2} U_{5}^{\prime \prime}+c_{3} \beta B_{2}^{2} U_{5}^{\prime \prime}+c_{3} \beta B_{1}=0 .
\end{aligned}
$$

Integration of (63) provides

$$
\begin{aligned}
c_{3} \alpha B_{1}^{2} U_{5}^{\prime \prime} & +\frac{3}{2} c_{3} \alpha B_{1}^{2} U_{5}^{\prime \prime 2}+c_{3} \alpha B_{1}^{2} U_{5}^{\prime 3}-c_{4} B_{1}^{2} U_{5}^{\prime}+c_{2} B_{1} B_{2} U_{5}^{\prime} \\
& +c_{3} \beta B_{2}^{2} U_{5}^{\prime}+c_{3} \beta B_{1} X_{1}=D
\end{aligned}
$$

where $D$ is constant of integration. It is clearly seen that equation (64) is difficult to solve.

3.6.2. If $a_{3}=0$ in Equation (59). Then, given characteristic equation is

$$
\frac{\mathrm{d} X}{a_{2}}=\frac{\mathrm{d} Y}{a_{1}}=\frac{\mathrm{d} U}{a_{4}} .
$$

It follows the similarity form as

$$
U(X, Y)=\frac{a_{4} Y}{a_{1}}+U_{6}\left(X_{6}\right),
$$

where $X_{6}=X-\left(a_{2} Y / a_{1}\right)$. From equations (66) and (58), we obtain the ODE as

$$
\begin{gathered}
a_{1} c_{3} \alpha U_{6}^{\prime \prime}+U_{6}^{\prime \prime}\left(3 a_{1} c_{3} \alpha\left(U_{6}^{\prime 2}+U_{6}^{\prime \prime}\right)-a_{1} c_{4}\right. \\
\left.+a_{2}\left(c_{2}+\frac{a_{2} c_{3} \beta}{a_{1}}\right)\right)=0 .
\end{gathered}
$$

By setting the particular values of arbitrary constants as $a_{1}=1, a_{2}=1, c_{1}=1, c_{2}=1, c_{3}=1, \alpha=1$, and $\beta=1$, the reduced form of ODE is

$$
U_{6}^{\prime \prime}+3 U_{6}^{\prime \prime} U_{6}^{\prime \prime}+3 U_{6}^{\prime 2} U_{6}^{\prime \prime}+U_{6}^{\prime \prime}=0,
$$

which on integration gives

$$
U_{6}^{\prime \prime}+\frac{3}{2} U_{6}^{\prime \prime 2}+U_{6}^{\prime 3}+U_{6}^{\prime}=C,
$$




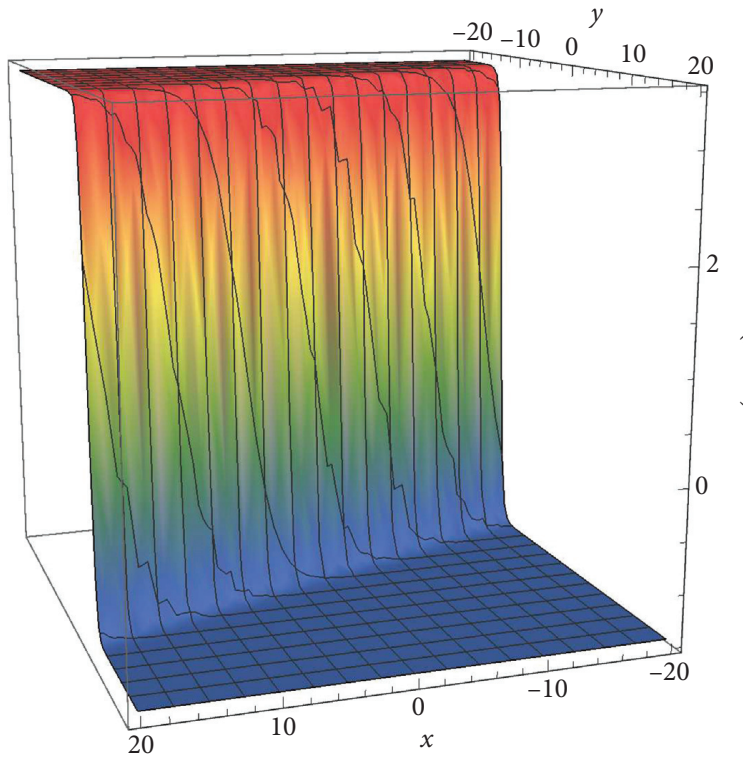

(a)

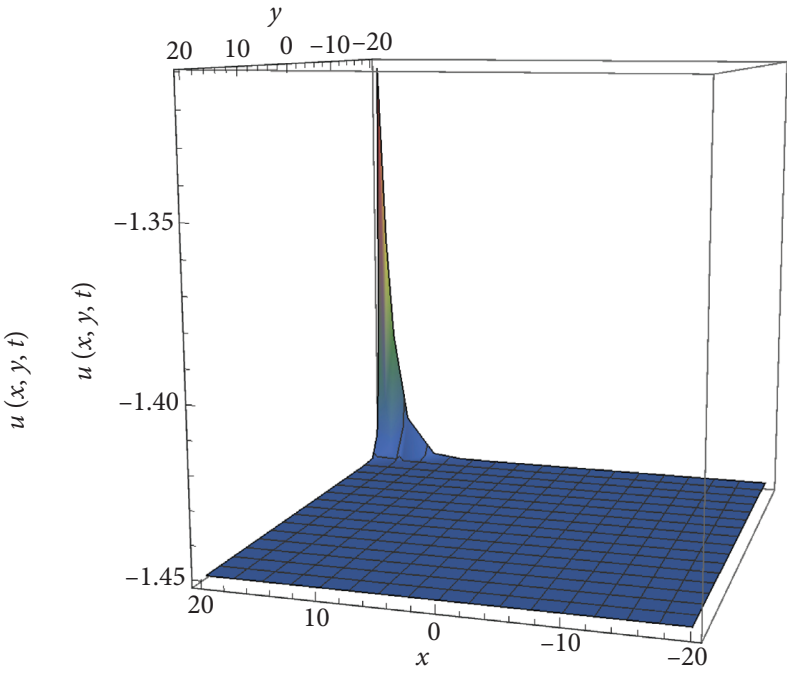

(b)

Figure 1: Three-dimensional shapes of annihilation of kink wave profiles for equation (3) at different values of $t$. (a) 3D profile of $u$ with $t=1$. (b) $3 \mathrm{D}$ profile of $u$ with $t=11$.

where $C$ is constant of integration. One of the particular solutions of equation (69) is found as

$$
U_{6}\left(X_{6}\right)=A_{0}+\frac{\left(\sqrt{[3]} 2\left(\sqrt{81 C^{2}+12}+9 C\right)^{(2 / 3)}-2 \sqrt{[3]} 3\right) X_{6}}{6^{(2 / 3)} \sqrt{[3]} \sqrt{81 C^{2}+12}+9 C}
$$

where $A_{0}$ is an arbitrary constant. Thus, the explicit exact solution of (2) is given as

$$
\begin{aligned}
u(x, y, t)= & \frac{\left(\sqrt{[3]} 2\left(\sqrt{81 C^{2}+12}+9 C\right)^{(2 / 3)}-2 \sqrt{[3]} 3\right)\left(a_{1}\left(c_{3} x-c_{4} t\right)+a_{2}\left(c_{2} t-c_{3} y\right)\right)}{6^{(2 / 3)} a_{1} c_{3} \sqrt{[3]} \sqrt{81 C^{2}+12}+9 C} \\
& +\frac{a_{4}\left(y-\left(c_{2} t / c_{3}\right)\right)}{a_{1}}+A_{0}+\frac{\int\left(y f_{1}(t)+f_{2}(t)\right) \mathrm{d} t}{c_{3}} .
\end{aligned}
$$

3.6.3. If $a_{3}=0$ and $a_{1}=0$. Then, Lagrange equation is

$$
\frac{\mathrm{d} X}{a_{2}}=\frac{\mathrm{d} Y}{0}=\frac{\mathrm{d} U}{a_{4}},
$$

which on solving proposes the following form of $U$ :

$$
U(X, Y)=\frac{a_{4} X}{a_{2}}+U_{7}\left(X_{7}\right),
$$

where $X_{7}=Y$. Substituting the value of (73) into (58), we get ODE as

$$
U_{7}^{\prime \prime}=0
$$

The solution of this ODE is

$$
U_{7}\left(X_{7}\right)=\alpha_{0} X_{7}+\alpha_{1}
$$

where $\alpha_{0}$ and $\alpha_{1}$ are arbitrary constants. Thus, from equations (75), (73), and (57), the analytic exact solution of (2) can be furnished as 


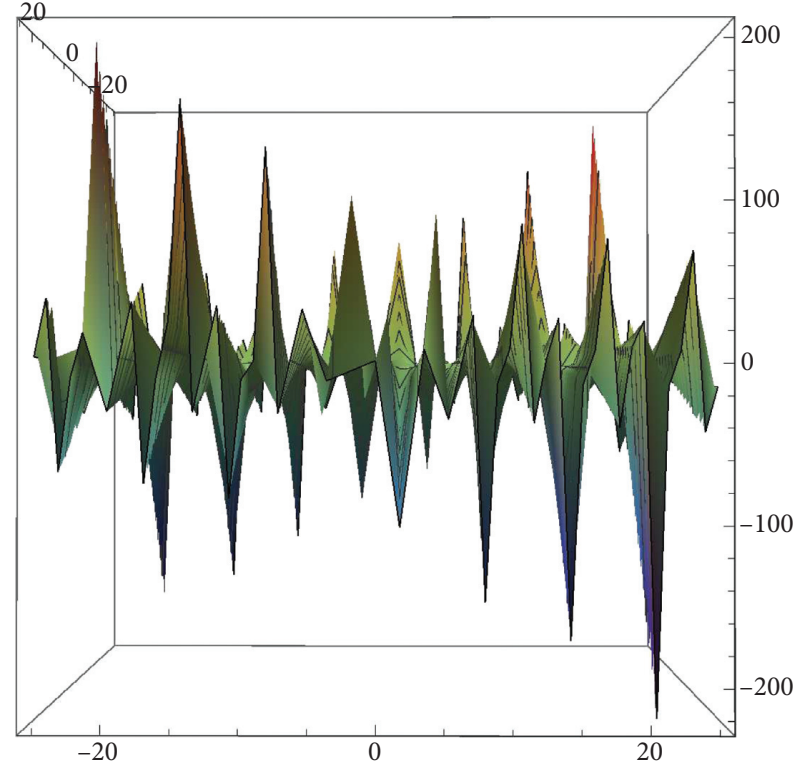

(a)

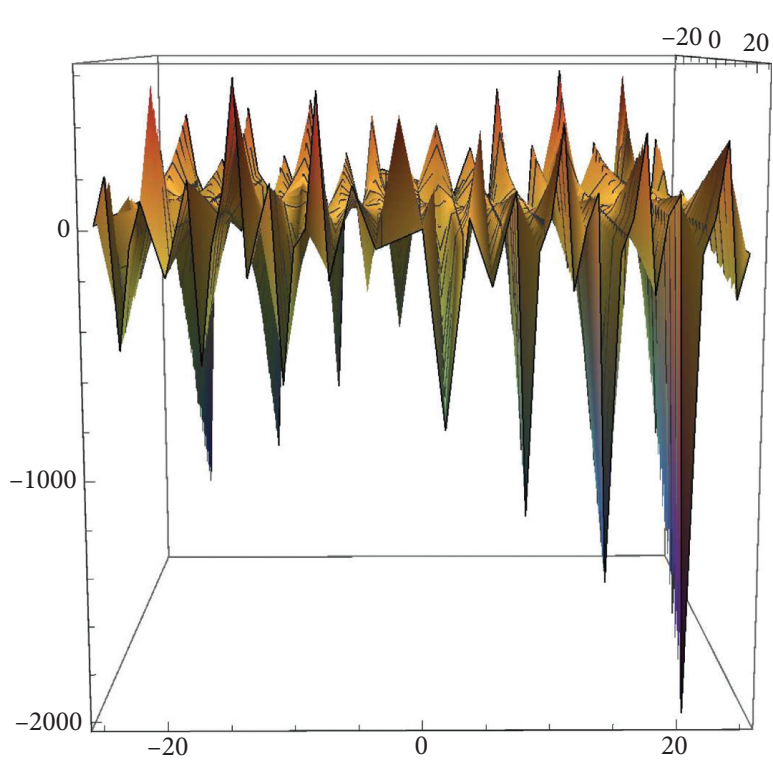

(b)

Figure 2: Three-dimensional shapes of periodic solitary waves for (35) with different values of $y$. (a) 3D profile of $u$ with $y=1$. (b) 3D profile of $u$ with $y=6$.

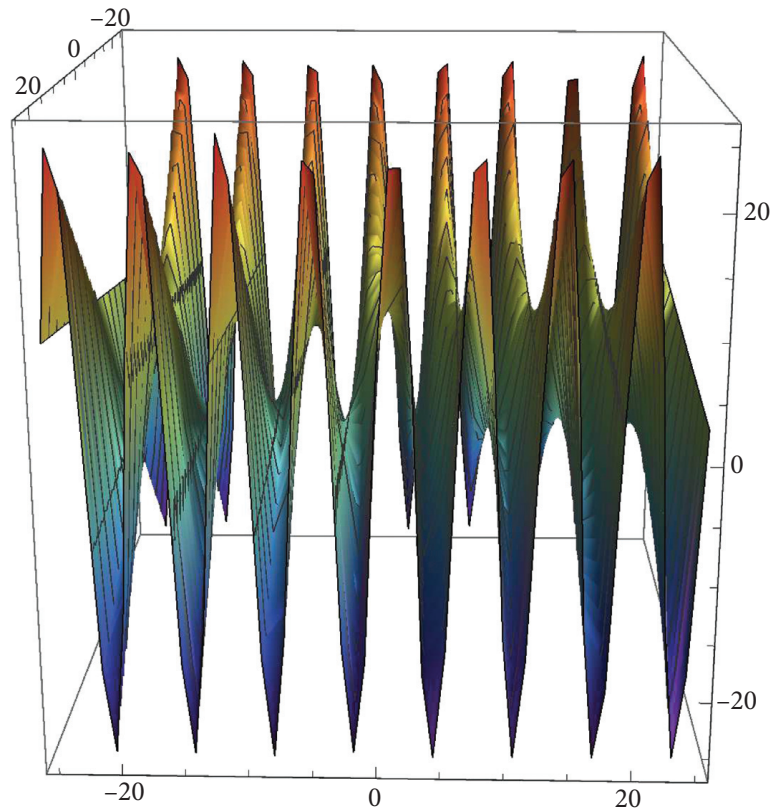

(a)

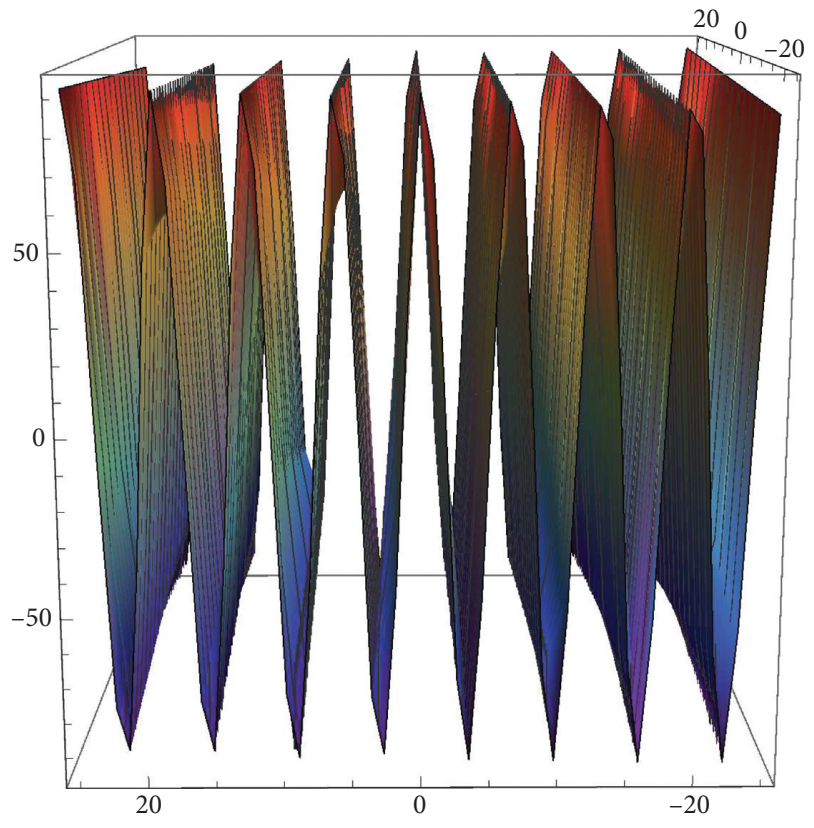

(b)

FIGURE 3: Three-dimensional shapes of oscillating solitary waves (multiple-front profiles) for (38) with different values of $y$. (a) 3D profile of $u$ with $y=2$. (b) $3 \mathrm{D}$ profile of $u$ with $y=9$. 


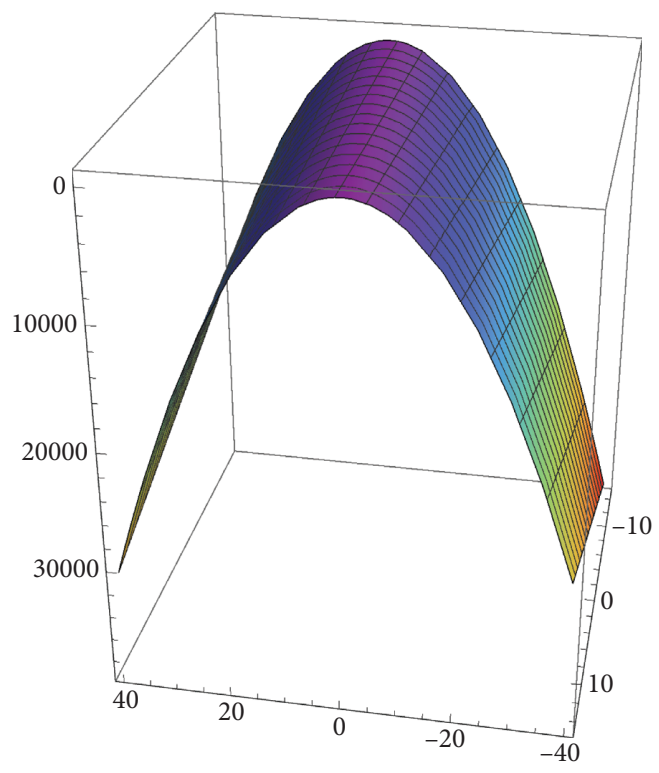

(a)

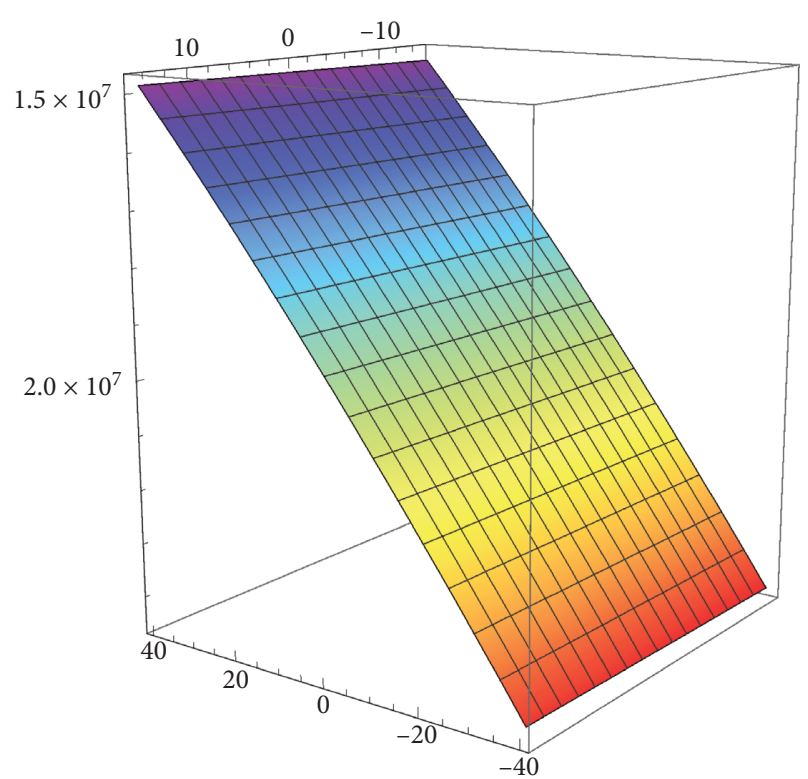

(b)

FIgURE 4: Three-dimensional shapes of annihilation of parabolic profile for equation (48) which gets annihilated at $t=20$. (a) $3 \mathrm{D}$ profile of $u$ with $t=2$. (b) $3 \mathrm{D}$ profile of $u$ with $t=20$.

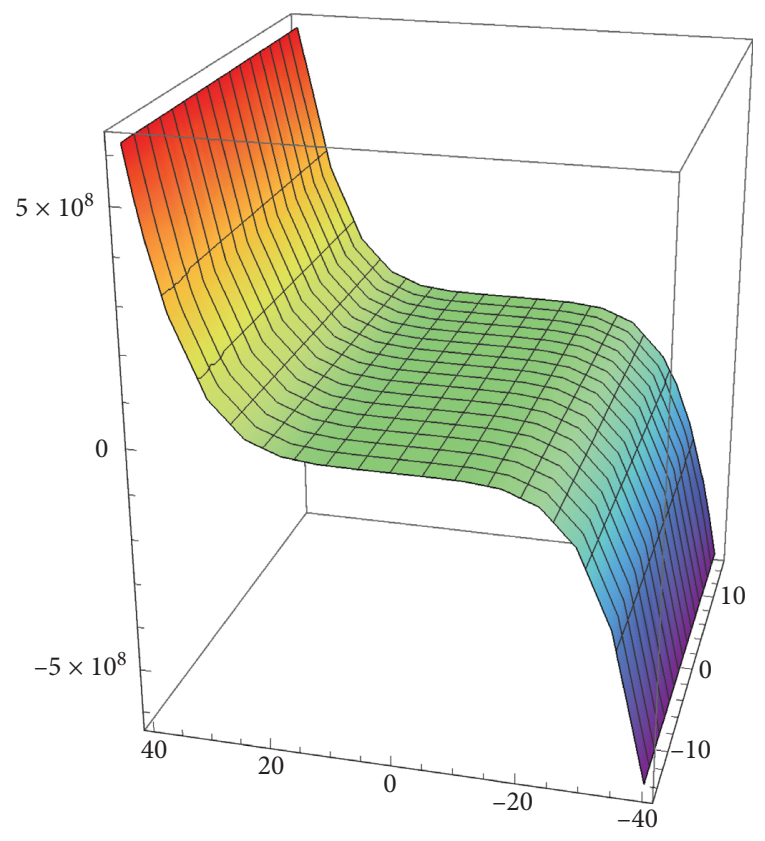

(a)

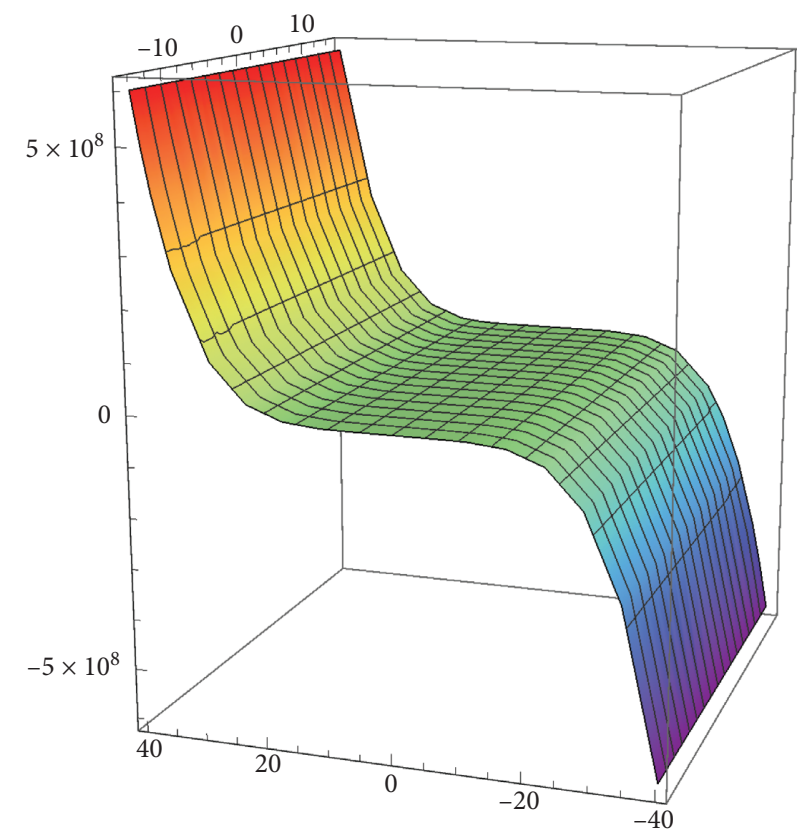

(b)

FIGURE 5: Three-dimensional shapes of asymptotic wave structures of equation (50) with different values of $y$. (a) 3D profile of $u$ with $y=1$. (b) 3D profile of $u$ with $y=20$. 


$$
u(x, y, t)=\alpha_{1}+\frac{-\left(\alpha_{0} a_{2} c_{2}+a_{4} c_{4}\right) t+a_{4} c_{3} x}{a_{2} c_{3}}+\alpha_{0} y+\frac{\int\left(y f_{1}(t)+f_{2}(t)\right) \mathrm{d} t}{c_{3}} .
$$

\section{Analysis and Discussion}

In the present section, a discussion about graphical structures of solutions is carried out. Since mathematical expressions do not produce the wave propagation of flow, we need physical as well as dynamical analysis of these solutions. Here, explicit analytic solutions of the STO equation (2) has been given by equations (3), (25), (32), (35), (38), (48), (50), (55), (71), and (76). All the obtained solutions contain arbitrary functions as well as constants. Therefore, by taking the adequate adjustment of these functions and constants, then we have analyzed the dynamical characteristics of obtained results in the following manner.

Figure 1 represents the physical nature of solution (3) with time. Kink nature of the solution is obtained by taking the values of constants as $c_{5}=1, c_{6}=1, \alpha=1$, and $\beta=1$. This profile is traced at $t=1$, for $-20 \leq x \leq 20,-20 \leq y \leq 20$. The kink solution is a localized solution, i.e., $u$ tends to a finite number when $t \longrightarrow \infty$. It was observed by numerical simulation that the behavior of exiting wave depends on time variation. It turns into single solitons at $t=11$ and is then annihilated after $t=16$.

Figure 2 demonstrates periodic solitary wave profiles of $u$ given by equation (35). The choice of arbitrary functions is taken as $f_{1}(t)=\tan (t), h_{1}(t)=\cot \left(b_{0} t+b_{1}\right), h_{2}(t)=\sin (t)$, and arbitrary constants as $b_{0}=5, b_{1}=10$, and $\beta=4$ for space range $-25 \leq x \leq 25,-25 \leq t \leq 25$. In Figure $2(\mathrm{~b})$, the corresponding $3 \mathrm{D}$ plot is shown for $y=6$. It was observed that periodicity decreases as the value of $y$ increases. This nonlinear behavior of soliton solution generally reveals the intersection of Riemann wave and long wave.

Figure 3 interprets the multiple-front solitary wave solution profile of $u$ given by equation (38) at $y=2$, for range $-25 \leq x \leq 25,-25 \leq t \leq 25$. The choice of arbitrary functions is taken as $h_{1}(t)=\cos (t), h_{2}(t)=\left(\tan \left(t^{2}+t+1\right) /(2 t+1)\right)$, $f_{2}(t)=\sin (t)$, and parameter $\beta=-0.5$. Initially, wave profile reveals curve-front wave nature of solution which gets converted into plane-front wave as $y$ increases.

Figure 4 shows the parabolic profile for the solution given by equation (48). Solution profile is observed at $t=2$, for $-15 \leq x \leq 15,-40 \leq y \leq 40$, and the values of arbitrary constants are taken as $A_{1}=0.110, A_{3}=0.115, c_{1}=2$, $c_{2}=0.001, c_{3}=1, c_{4}=-0.12, A=3, B=1, \beta=1, b_{0}=10$, and $b_{1}=0.91$, and the choice of arbitrary functions is taken as $f_{1}(t)=b_{0} t+b_{1}$ and $f_{2}(t)=\left(\left(2 b_{0} t+b_{1}\right) /\left(\tan \left(b_{0} t^{2}+\right.\right.\right.$ $\left.\left.\left.b_{1} t+5\right)\right)\right)$. The nonlinearity of solution (48) is annihilated at $t=20$. This parabolic profile of solution can be utilized in making the design of ballistic missiles.

Figure 5 is plotted between spatial and temporal axes $x-t$ which represent the asymptotic wave profile for solution (50) and is illustrated at $y=1$, for $-15 \leq x \leq 15$, $-40 \leq t \leq 40$. The values of constants are taken as $A_{1}=0.110$, $A_{3}=1.019, \quad c_{1}=2, \quad c_{2}=0.001, \quad c_{3}=1, \quad c_{4}=0.12, \quad \beta=1$, $b_{0}=10$, and $b_{1}=0.91$, and those of functions are taken as $f_{1}(t)=\left(\left(2 b_{0} t+b_{1}\right) /\left(b_{0} t^{2}+b_{1} t+5\right)\right)$ and $f_{2}(t)=\tanh (t)$.

\section{Conclusion}

In this research work, we have obtained some closed-form explicit solutions for the $(2+1)$-dimensional Sharma-Tasso-Olver (STO) equation by means of Lie approach, which are given by equations (3), (25), (32), (35), (38), (48), (50), (55), (71), and (76). These achieved solutions are entirely distinct from the earlier findings [19]. Also, these reported results are revealed to be more general because it contains arbitrary constants and independent functions $f_{1}(t), f_{2}(t), h_{1}(t)$, and $h_{2}(t)$. All the generated results are analyzed physically through Figures 1, 2, 3, 4, and 5 which shows parabolic, periodic solitary waves, oscillating traveling waves, kink wave profiles, etc. The dynamics of solitary wave profiles are vividly exhibited via Mathematica. This research shows that the Lie transformation method can be extended for obtaining explicit exact-analytic solutions of many other NLEEs that are emerging in optical physics, plasma physics, chemical physics, acoustics, etc. In summary, the proficient Lie symmetry technique that we implemented is robust, competent, and efficient to solve analytically.

\section{Data Availability}

All data used to support the findings of this study are included within the article.

\section{Conflicts of Interest}

The authors declare that there are no conflicts of interest regarding the publication of this paper.

\section{References}

[1] A.-M. Wazwaz, Partial Differential Equations and Solitary Wave Theory, Springer, New York, NY, USA, 2009.

[2] P. Devi and K. Singh, "Classical Lie symmetries and similarity reductions of the $(2+1)$-dimensional dispersive long wave system," AEJM-journal, vol. 14, 2020.

[3] S. Kumar and S. Rani, "Lie symmetry reductions and dynamics of soliton solutions of $(2+1)$-dimensional Pavlov equation," Pramana-Journal of Physics, vol. 94, p. 116, 2020.

[4] E. Fan and H. Zhang, "A note on the homogeneous balance method," Physics Letters A, vol. 246, no. 5, pp. 403-406, 1998.

[5] S.-J. Yu et al., J. Phys. A. 31, 3337 (1998).

[6] W. X. Ma, Z. Qin, and X. Lü, "Lump solutions to dimensionally reduced $\$ \$ \backslash$ varvec $\{\mathrm{p}\} \$ \$ \mathrm{p}-\mathrm{gKP}$ and $\$ \$ \backslash$ varvec $\{\mathrm{p}\} \$ \mathrm{p}$ -gBKP equations," Nonlinear Dynamics, vol. 84, no. 2, pp. 923-931, 2016.

[7] J.-G. Liu, Y.-Z. Li, and G. M. Wei, "Extended generalized hyperbolic-function method and new exact solutions of the 
generalized hamiltonian and NNV equations by the symbolic computation," Chinese Physics Letters, vol. 23, p. 1670, 2006.

[8] M. Wadati, H. Sanuki, and K. Konno, "Relationships among inverse method, Backlund transformation and an infinite number of conservation laws," Progress of Theoretical Physics, vol. 53, no. 2, pp. 419-436, 1975.

[9] W. Hong and Y.-D. Jung, "Auto-Bäcklund transformation and analytic solutions for general variable-coefficient $\mathrm{KdV}$ equation," Physics Letters A, vol. 257, no. 3-4, pp. 149-152, 1999.

[10] Z. Sheng, "The periodic wave solutions for the $(2+1)$-dimensional Konopelchenko-Dubrovsky equations," Chaos, Solitons \& Fractals, vol. 30, no. 5, pp. 1213-1220, 2006.

[11] Y. C. Hon and E. Fan, "Uniformly constructing finite-band solutions for a family of derivative nonlinear Schrödinger equations," Chaos, Solitons \& Fractals, vol. 24, no. 4, pp. 1087-1096, 2005.

[12] W.-X. Ma and M. Chen, "Do symmetry constraints yield exact solutions?" Chaos, Solitons \& Fractals, vol. 32, no. 4, pp. 1513-1517, 2007.

[13] Z. J. Lian and S. Y. Lou, "Symmetries and exact solutions of the Sharma-Tass-Olver equation," Nonlinear Analysis, vol. 63, p. 1167, 2005.

[14] Z. Yan, MM Research. 22, 302 (2003)..

[15] Y. Uğurlu and D. Kaya, Phys. Lett. A. 370, 251 (2007).

[16] A.-M. Wazwaz, "New solitons and kinks solutions to the Sharma-Tasso-Olver equation," Applied Mathematics and Computation, vol. 188, no. 2, pp. 1205-1213, 2007.

[17] L. Jianming, D. Jie, and Y. Wenjun, "Solitary wave solutions for the boussinesq and fisher equations by the modified simple equation method," Mathematical Letters, vol. 2, p. 8, Article ID 935710, 2011.

[18] S. Wang, X.-y. Tang, and S.-Y. Lou, "Soliton fission and fusion: burgers equation and Sharma-Tasso-Olver equation," Chaos, Solitons \& Fractals, vol. 21, no. 1, pp. 231-239, 2004.

[19] B. Ren and W.-X. Ma, "Rational solutions of a $(2+1)$-dimensional Sharma-Tasso-Olver equation," Chinese Journal of Physics, vol. 60, pp. 153-157, 2019.

[20] G. W. Bluman and J. D. Cole, Similarity Methods for Differential Equation, Springer-Verlag, New York, NY, USA, 1974.

[21] P. J. Olver, Applications of Lie Groups to Differential Equations, Springer-Verlag, New York, NY, USA, 1993.

[22] D. Kumar and S. Kumar, "Some new periodic solitary wave solutions of (3+1)-dimensional generalized shallow water wave equation by Lie symmetry approach," Computers \& Mathematics with Applications, vol. 78, no. 3, pp. 857-877, 2019.

[23] M. Kumar and A. K. Tiwari, "Soliton solutions of BLMP equation by Lie symmetry approach," Computers \& Mathematics with Applications, vol. 75, no. 4, pp. 1434-1442, 2018.

[24] S. Kumar, M. Niwas, and A. M. Wazwaz, "Lie symmetry analysis, exact analytical solutions and dynamics of solitons for $(2+1)$-dimensional NNV equations," Physica Scripta, vol. 95, no. 9, Article ID 095204, 2020.

[25] S. Kumar and D. Kumar, "Lie symmetry analysis and dynamical structures of soliton solutions for the $(2+1)$-dimensional modified CBS equation," International Journal of Modern Physics B, vol. 34, no. 25, Article ID 2050221, 2020.

[26] S. Kumar and Y. K. Gupta, "Generalized invariant solutions for spherical symmetric non-conformally flat fluid distributions of embedding class one," International Journal of Theoretical Physics, vol. 53, no. 6, pp. 2041-2050, 2014.
[27] S. Kumar and A. Kumar, "Dynamical structures of solitons and some new types of exact solutions for the (2+1)-dimensional DJKM equation using Lie symmetry analysis," Modern Physics Letter B, vol. 34, 2020.

[28] P. Hydon, Symmetry Methods for Differential Equations, Cambridge University Press, Cambridge, UK, 2000. 